and 80 millimeters apart, they were felt as one; while at a corresponding locality on the right arm the skin was so sensitive that points but 17 millimeters apart could be felt as two. It must also be observed that this particular person, with reference to a large number of activities, had always previously been practically ambidextrous-indeed, he never had to favor the left arm at all. In his position as gymnasium instructor, he could (before the accident) manipulate the dumbbells, Indian clubs, play base-ball, hand-ball, and the like, with the left hand as well as with the right. It would seem, then, that the sensibility of the skin over the injured forearm was lost simply because that member was for so long a time immovable. This has an important bearing on the oft-mentioned principle to the effect that "the localizing power is delicate in proportion as the skin covers a movable part of the body."

\title{
ADJUSTMENT OF SIMPLE PSYCHOLOGICAL MEASUREMENTS.
}

BY E. W. SCRIPTURE, PH.D.,

Yale University.

Given the measurements $a_{1}, a_{2}, \ldots, a_{n}$, to find the best representative value.

The use of the arithmetic mean

$$
a=\frac{a_{1}+a_{2}+\ldots+a_{n}}{n}
$$

involves the assumption that the deviations from the average, $V_{1}=a_{1}-a, V_{2}=a_{2}-a, \ldots, V_{n}=a_{n}-a$, occur with probabilities according to Gauss's law,

$$
y=w_{0} \exp \left(-\pi w_{0}^{2} V_{\mathrm{s}}\right),
$$

where $y$ is the probability of any deviation $V$, and $w_{0}$ is the probability for $V=0$.

Bernoulli's theorem justifies the substitution of the actual frequencies for the probabilities when $n$ is very large, thus enabling us to test the law by actual experience. The law has been verified for careful measurements in physical and 
astronomical work. It does not hold good for statistical and psychological measurements.

The use of the geometric mean

$$
a=\sqrt[\pi]{a_{1} \times a_{2} \times \cdots \times a_{z}}
$$

involves the assumption that

$$
y=w_{0} \exp \left(-\pi w_{0}\left[\log a_{i}-\log a\right]\right) \text {. }
$$

This distribution of deviations has been approximately verified for statistical and psychological results.

As $n$ can be made large only on exceptional occasions, the choice of the representative value is thus left largely to the discretion of the investigator. To avoid this uncertainty Fechner has proposed the use of the median or central value, whose position in the series of separate results arranged according to size is given by

$$
\frac{a_{1}^{0}+a_{2}^{0}+\ldots+a_{n}^{0}+1}{2}=\frac{n+1}{2} .
$$

That is, if all the results are to be arranged in the order of their size, the median value will be just in the middle. Since with finite units of measurement there will be a number of results having the same value around the middle, the value $a$ will be determined by interpolation. The importance of the use of the median lies in the fact that it involves no assumption in regard to the distribution of the separate deviations. When the results follow Gauss's law the median is the same as the arithmetic mean; when their logarithms follow this law it is the same as the geometric mean. The arbitrary assumption of either law is thus excluded.

This method of adjustment, which seems logically the only correct one, has been employed for some time in my laboratory. The calculation of the median can often be performed mentally where that of the arithmetic mean requires pencil and paper and that of the geometric mean requires logarithms also. Cases have frequently arisen where the arithmetic mean unquestionably misrepresents the results, whereas I have never found any objection to the median that is not common to all representative values. 